\title{
Two-Dimensional Speckle Tracking Echocardiography: Clinical Usefulness
}

\author{
Prof. H. I. Lutfur Rahman Khan \\ Professor of Cardiology \& Ex- Head of the Department of Cardiology, \\ Dhaka Medical College, Dhaka
}

(Cardiovasc. j. 2018; 10(2): 111-112)

Traditionally, the assessment of left ventricular (LV) function has been made from an overall visual impression of $\mathrm{LV}$ wall motion noted on multiple views on $2 \mathrm{D}$ echocardiogram. Objective measurements such as fractional shortening and calculation of the ejection fraction $(\mathrm{EF})$ often provide only a global estimation of LV function and are subject to interobserver and intraobserver variability. Operator experience also influences the visual assessment of LV function.

Strain imaging, also called myocardial deformation imaging is a technique developed in an attempt to standardize regional assessment of LV mechanics. During recent years, deformation imaging (strain and strainrate imaging) called myocardial mechanics, have emerged as valuable tools for more comprehensive and reliable assessment of myocardial function. And this creates a new window to assess the myocardial ischemic insult by assessing the myocardial deformation.

Myocardial deformation study can be done by Tissue Doppler Imaging (TDI) and Speckle Tracking Echocardiography (STE) using two or three dimensional strain. Tissue Doppler has limitation of angle dependency. STE is a relatively new, angle and user-independent technique used for the evaluation of myocardial function.

Myocardial deformation is the change of shape (lengthening and shortening) of cardiac muscle in relation to original length during cardiac cycle. During the systolic phase, longitudinal motion occurs with the base moving towards apex is associated with inward movement of the ventricular walls and differential rotation of the apex and the base. The opposite events are seen in diastole. While in systole the base rotates clockwise, the apex rotates counterclockwise. Untwisting occurs in diastole with opposite movements of the apex (clockwise) and base (counterclockwise). Thus during ventricular function myocardial deformation occurs in several directions e.g. longitudinal, radial, circumferential, area change, rotational, and twisting. The indices of deformation measurement are displacement, velocity, strain, and strain rate.

Strain is a measure of myocardial deformation. It is defined as the change of myocardial fiber length at end-systole compared to its original length in relaxed state at end-diastole. It is a dimensionless index of change of length. Strain parameters are measured in longitudinal strain, radial strain, circumferential strain, area strain, rotational strain, twisting, torsion, and shear strain. Strain is calculated by subtracting the resting myocardial fiber length (Lo) from that at the end of systole (L) and dividing it by the resting length $(\mathrm{Lo})$ at end diastole. $=(\mathrm{L}-\mathrm{Lo}) / \mathrm{Lo}$. Strain can be negative, when end systolic dimension decreases in comparison to end diastolic dimension, and again it can be positive also, when end systolic dimension increases in comparison to end-diastolic dimension.

Longitudinal strain represents shortening of the left ventricular length from base to apex during systole and is usually measured in any of the long-axis views. As there is a decrease in the

Address of Correspondence : Prof. Dr. H. I. Lutfur Rahman Khan, Professor of Cardiology \& Ex- Head of the Department of Cardiology, Dhaka Medical College, Dhaka. 
longitudinal length, it is denoted by a negative (-) sign. Longitudinal strain increases from base $(-15 \%)$ to apex $(-20 \%)$. Lower limit of $-18.5 \%$ may be appropriate for global longitudinal strain in normal subjects.

Radial or transverse strain refers to thickening of the LV wall during systole. Since wall thickness normally increases during systole, the measured strain is a positive value. It is typically measured in parasternal short-axis basal and apical views. Radial strain is usually more than 44.5\% in normal subjects Circumferential strain refers to the change in the circumference of each segment, and is measured using kernels placed along the circumference of the myocardium in parasternal short-axis views. It has a negative value as the circumferential dimension decreases at end systole. In normal subjects, it increases from base through apex $(-28 \%$ to $-33 \%$ to $-35 \%$ from base to mid to apex).

In the normal individual, the apex rotates more than the base. With LV contraction, the cardiac apex and the base move in counterclockwise and clockwise directions, respectively, when viewed from apex. By convention, counter clock wise motion is assigned a positive (+) sign and clockwise motion a negative (-) sign. "Twist" is calculated as the algebric difference in the rotation of the apex and base; in other words, how far apart a point in the apex and base move from each other. Twist = Apex $-(-$ Base $)$. In normal subjects, apical rotation is 60 and basal rotation is -6o, producing a twist of 120 . "Torsion" is a way of adjusting for the distance of a point of interest from the reference point in the base, as it is expected that the twist will be higher for the segments farther away from the base.

Decrease in peak strain usually indicates segmental dysfunction. Analysis of time to peak strain in various segments can be used to measure ventricular dyssynchrony. Segmental variation in time to peak strain may represent regional dyssynchrony and can be used to predict response and assist in cardiac resynchronization therapy (CRT).

The strain rate is the myocardial deformation rate (i.e. how fast the deformation occurs). It is expressed as seconds-1. It is less dependent on LV load variations than strain. Echocardiographically strain analysis can be done by TDI and Speckle Tracing Echocardiography (STE).

Speckle tracing echocardiography (STE) is a novel technique of cardiac imaging for quantifying complex cardiac lesion based on frame to frame tracking of ultrasonic speckles in grey scale images. STE is relatively angle independent technology that can measure global and regional strain, strain rate, displacement, and velocity in longitudinal, radial and circumferential directions. It can also quantify rotational movements such as rotation, twist, torsion and shear of the myocardium.

Two-dimensional STE has been found clinically useful in the assessment of cardiac systolic function including detection of myocardial ischemia and diastolic function as well as providing new insights in deciphering cardiac physiology and mechanics in cardiomyopathies, and identifying early subclinical changes in various pathologies. A large number of studies have evaluated the role of 2D STE in predicting response to cardiac resynchronization therapy in patients with severe heart failure. Emerging areas of application include prediction of myocardial viability, stress cardiomyopathy, restrictive cardiomyopathy, constrictive pericarditis, detection of rejection and coronary stenosis in heart transplant patients, early detection of chemotherapy induced cardiotoxicity in patients receiving chemotherapy for cancer, in valvular heart disease, congenital heart disease, and effect of intracoronary injection of bone marrow stem cells on left ventricular function in patients with acute myocardial infarction.

Myocardial deformation imaging or strain imaging allows objective evaluation of myocardial mechanical function. 2D STE overcomes several shortcomings of TDI and demonstrate enormous potential for clinical use. The emerging technique of three-dimensional (3D) STE may further extend its clinical usefulness. 